\title{
Bringt die Qualitätsoffensive der Kliniken einen durchschlagenden Erfolg? Die Krux mit den Qualitätsberichten
}

Bald ist es wieder so weit: Am 30. November müssen die gut 2000 deutschen Kliniken zum zweiten Mal ihre Qualitätsberichte im Internet veröffentlichen. Doch ähnlich wie bei der ersten gesetzlich vorgeschriebenen Veröffentlichung im Herbst 2005 erwarten viele Experten von den Daten nur einen begrenzten Nutzen. Der Gemeinsame Bundesausschuss und die Deutsche Krankenhausgesellschaft dagegen sind optimistisch, dass die Maßnahmen, die sie zur Sicherung der Qualität der Berichte eingeleitet haben, greifen werden.

Die gesetzlich vorgeschriebene Veröffentlichung von Qualitätsberichten soll dazu beitragen, die Qualität der Kliniken und der angebotenen Leistungen zwischen den Häusern transparent und vergleichbar zu machen. Doch schon darüber, was eine hohe Qualität ausmacht, scheiden sich die Geister. So müssen hohe Fallzahlen nicht unbedingt für ein Haus sprechen. Schon ein schlechter Chirurg reicht aus, um den Schnitt auch in einer großen Klinik kaputt zu machen.

Ein weiteres Manko ist, dass bei der Erstellung der Berichte keine aktuellen Daten aus dem laufenden Jahr mit in die Bewertung eingehen. Was also Ende November zu lesen sein wird, bezieht sich auf das Jahr 2006, sodass die Daten und Ergebnisse unter Umständen schon am Tage der Veröffentlichung überholt sein könnten. Und selbst wenn die Qualitätsberichte gut aufbereitet sind, sind viele Zuweiser, aber vor allem die Patienten überfordert, wenn es darum geht, die Qualität einzelner Häuser oder der angebotenen Leistungen zu vergleichen. Denn eine vergleichende Analyse wird es auch in diesem Jahr nicht geben.

\section{GBA und DGK haben die Initiative ergriffen}

Zwar haben die Mitarbeiter des Gemeinsamen Bundesausschusses (GBA) in den vergangenen zwei Jahren versucht, die Transparenz und die Qualität der Berichte zu verbessern. So gibt es jetzt unter anderem differenzierte Ausfüllhinweise, eine Lesehilfe für die Nutzer, und die Angaben zu den Maßnahmen für das
Qualitätsmanagement sollen stärker systematisiert werden. Gefordert wird zudem eine einheitlichere Verwendung medizinischer Fachtermini.

Und auch die Kliniken geben sich selbstbewusst. Denn die Deutsche Krankenhausgesellschaft (DGK) hat sich Mitte des Jahres mit den Krankenkassen darauf verständigt, dass 27 Qualitätsindikatoren für die Veröffentlichung im Qualitätsbericht verpflichtend sind, um die Vergleichbarkeit der Behandlungsergebnisse gewährleisten zu können. Darüber hinaus steht es dem Krankenhaus frei, weitere bewertete Indikatoren zu publizieren. Insgesamt können die Kliniken auf mehr als 200 Qualitätsindikatoren zugreifen und ihr Abschneiden im bundesweiten Vergleich darlegen. Grundlage sind die Daten, die alle Krankenhäuser an die Bundesgeschäftsstelle Qualitätssicherung (BQS) übermitteln. Die DGK zumindest sieht hier einen „bahnbrechenden Forschritt in der Transparenz und Qualität der stationären Versorgung“.

Ob sich all dies in einer besseren Vergleichbarkeit der Daten niederschlagen wird, wird sich allerdings erst zeigen, wenn die neuen Qualitätsberichte veröffentlicht worden sind.

\section{Klinikführer: Mal besser, mal schlechter}

Inzwischen gibt es viele - mehr oder weniger umfangreiche und subjektive Angebote, die Patienten bei der Suche nach der für sie optimalen Klinik unterstützen wollen. Allein auf Fallzahlen und Patientenberichten basiert zum
Beispiel Medmonitor. Dieses private Portal ist seit Mai dieses Jahres online, enthält bisher aber nur sehr wenige Beurteilungen.

Etwas mehr Vergleichsmöglichkeiten bietet ein Klinikführer der Techniker Krankenkasse. Für diesen gaben 100000 Versicherte Auskunft über ihre Zufriedenheit mit dem Behandlungsergebnis, der medizinisch-pflegerischen Versorgung oder der Organisation und Unterbringung. Ergänzt werden die Daten mit Angaben über die Ausstattung der Krankenhäuser oder der behandelten Fallzahlen. Sicherlich ist dies ein guter Ansatz, allerdings werden nur rund ein Zehntel aller deutschen Kliniken erfasst.

Deutlich umfassender ist das Angebot der Stuttgarter AnyCare GmbH, die derzeit zusammen mit der Abteilung Klinische Sozialmedizin des Universitätsklinikums Heidelberg ihr Angebot „QualitätsKompass Klinik“ um ein gemeinsames wissenschaftliches Projekt, die „Ärztebefragung 2007“, erweitert.

Im Rahmen der bundesweiten, unabhängigen Studie erhalten alle in neun Metropolregionen - eingeschlossen sind derzeit zum Beispiel Stuttgart, Berlin, Frankfurt oder München - ansässigen Haus- und Fachärzte aus insgesamt 18 Fachdisziplinen einen Fragenkatalog. Dabei können die befragten Ärzte ihre Empfehlung für eine Klinik in ihrer Region abgeben. Gefragt wird aber auch, wie sie die medizinische Kompetenz einer Fachabteilung oder die praktische Zusammenarbeit mit den Niedergelassenen einschätzen.

Immerhin erreicht das Projekt so über $50 \%$ der niedergelassenen Ärzte - und damit auch die Hälfte der Bevölkerung in Deutschland. Neben den Patienten profitieren auch die Kliniken. Denn sie können erfahren, wo sie aus Sicht der niedergelassenen Ärzte vor Ort stehen. Die ersten Ergebnisse werden voraussichtlich Ende November vorliegen. sts 